\title{
Information and Announcements
}

\section{Admission to PhD Programme in Science Education-2020*}

\author{
Homi Bhabha Centre for Science Education \\ Tata Institute of Fundamental Research (A Deemed University)
}

Applications are invited from students/science and mathematics teachers/educators for admission to the PhD Programme in Science Education at Homi Bhabha Centre for Science Education, Tata Institute of Fundamental Research, V. N. Purav Marg, Mankhurd, Mumbai - 400088, INDIA.

\section{Eligibility}

M.Sc. (in any subject), M.E., M.Tech. or a Master's degree (M.A./ M.S.W.) in any of the social/ behavioural sciences/ psychology/ linguistics/ sociology/ economics/ anthropology, M.Ed. with a science or social sciences/ humanities undergraduate degree.

\section{Scholarships}

Rs.31000/- per month till registration, Rs.35000/- per month post-registration Additionally, 24\% HRA in lieu of on-campus institute accommodation Annual contingency grant of Rs.40000/-

\section{Important Dates}

Application submission deadline: March 31, 2020

Written test: May 10, 2020

Interview: June 3rd week, 2020

To apply online, to download the form and for more information about admission please visit: http://wwW.hbcse.tifr.res.in/admissions/

For information about the PhD Programme please visit: http://www.hbcse.tifr.res.in/graduate-school

For details on areas of research please visit:

http://www.hbcse.tifr.res.in/research-development

For more information, contact:

Phones: (022) 25072304/25072230; Fax: (022) 25566803; Email: admissions@hbcse.tifr.res.in 